\title{
Letter to the editor: Therapeutic misconception and the role of the Research Ethics Committee
}

\author{
Akira Akabayashi*,1 \\ ${ }^{1}$ Department of Biomedical Ethics, Faculty of Medicine University of Tokyo, Tokyo, Japan; Division of Medical Ethics, New York \\ University School of Medicine, NY, USA \\ *Author for correspondence: Tel.: +1 8135841 3511; Fax: +1 8135841 3319; akirasan-tky@umin.ac.jp
}

First draft submitted: 29 November 2018; Accepted for publication: 20 June 2019; Published online: 23 August 2019

Keywords: informed consent $\bullet$ Japan $\bullet$ research ethics committee $\bullet$ research ethics consultation $\bullet$ therapeutic misconception

A commentary by Takashima et al. [1] reported on the reviewing process applied to the trial for clinical application of the world's first-in-human induced pluripotent stem cell-derived products to age-related macular degeneration (AMD). I raise several comments about this article from the perspective of research ethics. As safety validation is not an expertise of mine, I only question the authors' understandings of ethics committees and therapeutic misconception.

\section{Therapeutic misconception}

On page 125, line 12, the authors state, "She (Dr Takahashi) carefully explained that the clinical trial was an early (sic) phase trial to avoid unrealistic expectations," citing reference 8 . There, in the session chaired by Dr Muto, the last author of the article, Dr Takahashi reportedly said the following to the patients:

"The main objective of this clinical study is to consider safety. But as a secondary objective, we also expect efficacy. As stated earlier, it is not that we increase eyesight dramatically, but that we make it brighter with less distortions, or increase the sensitivity of the retina. Even if eyesight does not increase by that much, if sensitivity increases, things in general become brighter. We plan to confirm these aspects in this study."

(translated by a professional translating company, underlines mine)

I showed the original Japanese description to two professional Japanese language teachers. I asked them, "By adding 'But' and 'also' to the researchers' own enthusiasm and expectations for success, would the patients feel as if the trial will be more beneficial to them than if the 'But,' 'also,' and enthusiasm had not been included?' Both teachers agreed with this statement. They further suggested that, in order to be more objective, the researchers should have used 'And' instead of the 'But', delete 'also expect efficacy,' and instead, simply state the scientific facts.

Granted, Dr Takahashi did state, “. . it is not that we increase eyesight dramatically." However, I surmise that the rest of this long statement should be a simple statement of the purely scientific expectations, such as 'It is possible that sensitivity may increase, but we have no scientific evidence on this yet.' My strong assumption is that these dialogues with patients were highly likely to cause therapeutic misconception. It is easy for patients to understand that the trial will not increase eyesight dramatically, and conveying that information is not difficult either. Avoiding therapeutic misconception, however, requires careful thought and consideration from different dimensions, as well as comprehending all contextual cues; the first and last author of this paper do not seem to understand these.

On an even more serious note, on line 17, the authors write: "In the end, the institutional review boards (IRBs) accepted the researchers' proposal to describe the potential therapeutic benefits of the trial as being less than what was in fact expected." This goes entirely against the informed consent doctrine of research ethics, namely not telling the truth. The authors should disclose both the informed consent that was recommended by the IRB as well as the one that was actually used in the study. It is easy to expect from the context that the one used lessened the expected benefits. However, in my opinion, the serious ethical issue created by this is that the truth was not stated in the informed consent form. This is a serious breach of the research ethics principle. 


\section{Role of the committee $\&$ author recommendations}

It seems that the authors tried to make recommendations for the future review process on page 126 . However, these are either unclear or just out of focus.

In the first part, the authors state (or just reiterate), “... it is crucial to inform participants of both the risks and benefits in a detailed and accurate manner," citing King's old argument in 2000 (reference 15). The authors proposed nothing new to improve the informed consent process.

Second, I take issue with the first running statement under the title: "when informing participants of potential direct therapeutic benefits, the committees should identify whether the participants can make reasonable choices free of therapeutic misconception." Is this truly the committee's responsibility?

At the very most, the committee might be able to check the informed consent explanatory documents. By 'the committees should identify whether the participants can make reasonable choices free of therapeutic misconception,' do the authors mean that the committee should meet with each of the participants? The term 'identify' is incredibly vague here, and the sentence provides no concrete measure or suggestion as to what the committee can or should do.

In addition, on line 33, the authors present a criticism, stating that "no committee discussed the provision of special assistance to participation with AMD." While I would agree that this is partially the responsibility of the committee, I believe that this falls on the research ethics consultation team. The committee cannot understand the real minutes of the actual clinical settings. As I see it, the committee is established to point out major problems and general ideas to improve informed consent, while the research ethics consultation team, working directly with researchers in clinical settings, would help ensure the appropriate execution of the informed consent process by the researchers.

I do not know how many of the authors have actual experience in chairing ethics committees, but this article provides hardly any clinical implications or other measures that could improve the running of an ethics committee.

\section{Clarify the statement made by the Japanese government}

Recently, the Japanese government has been severely criticized by Nature Editorial and News [2,3] for its hasty attitude toward regenerative medicine regarding fast-track-approval. In this regard, there is a confusing sentence on page 124, line 21. "News of the trial attracted great public attention including that of patients, the public, industry, academia and the Japanese government, which implied that any factor leading to failure would not be tolerated." Since I could not understand the sentence fully, I asked 10 native English speakers, "Would you interpret this sentence to mean that the Japanese government (as a part of the listed group) implied that any factor leading to failure would not be tolerated?" Eight agreed and two said that they could not understand what the authors meant to say. As the AMD trial is supported by governmental top-down public funding, if the government did in fact imply to the researchers that failure is not tolerated for this research study, then this is not justifiable conduct on the government's part. However, for the government to state that it 'expects' success would be ethically acceptable. Of course, the sentence by Takashima et al. is not pointing to the government alone, but other players as well. My main point is whether the government was involved in any way in promoting this environment. I would ask that the authors clarify what was actually said by the government to the researchers in this instance. If the government did in fact tell the researchers that they were not allowed to fail, then this represents significant misconduct by the government. Either way, the authors bear the responsibility to clarify the truth.

Notably, if the authors were to come back and clarify that this was not what the government stated, they must understand that they have already jeopardized the credibility of the Japanese government significantly, by publishing that unclear and potentially misleading sentence in an influential international journal. Thus, while not all wrongs can be righted here, I urge the authors to explain their intended meaning if they did not mean to criticize the government.

\section{Future perspective}

It is clear that the responsibility and burden shouldered by research ethics committees are increasing substantially. All the internal committee members are serving on these committees voluntarily, and external committee members receive minimal remuneration. My criticism is that the authors are imposing an extra burden on the research ethics committee and not seeing the reality. The most effective recommendation, I believe, would be to establish infrastructure (including personnel such as Certified IRB Professionals in the USA) for research ethics consultations - specifically, an office of human research support which also provides ethical support at each institution in Japan - as soon as possible. Some of the functions of 'research ethics consultations' in the Japanese context, in my 
view, include: help researchers write appropriate (both scientifically and ethically) application for committee review in advance; help researchers write informed consent forms which will be easy to understand for subjects; tutor researchers and clinical coordinators on how to properly obtain informed consent in clinical settings, avoiding therapeutic misconceptions or coercion; and support researchers on any other ethics-related issues while the trial is ongoing.

Although this would require a substantial initial investment, it will contribute to sound and sustainable research progress in the future. In fact, such a training project was initiated in 2016 supported by the Japan Agency for Medical Research and Development, but the number of trainees is lacking and the quality of those trained under the project is still uncertain.

Finally, from the conflicts of interest disclosure statement in the article, the authors are supported by public funding. More concrete, practical recommendations from the authors would be greatly helpful for ethics committees and will help avoid therapeutic misconceptions.

\section{Conclusion}

A proposal has been made to establish 'research ethics consultation' in the Japanese context, which involves an initial check of protocols for scientific and ethical integrity before ethics committee review, helping researchers draft appropriate informed consent forms, providing guidance on the clinical practice of the informed consent process in order to avoid therapeutic misconception and coercion, and troubleshooting all ethics-related problems during clinical trials.

\section{Author contributions}

Conceptualization, writing - original draft, writing - review \& editing

\section{Financial \& competing interests disclosure}

A Akabayashi is President of the Japan Association for Bioethics (JAB) and chair of research ethics committee of The University of Tokyo Faculty of Medicine; this paper reflects the author's personal academic analyses and opinions and does not represent JAB's or University of Tokyo's official position. The author has received grants from The Hitachi Global Foundation and the Kurata Grant, No. 1373. The authors have no other relevant affiliations or financial involvement with any organization or entity with a financial interest in or financial conflict with the subject matter or materials discussed in the manuscript apart from those disclosed.

No writing assistance was utilized in the production of this manuscript except the italicized part.

\section{Disclaimer}

The opinions expressed in this interview are those of the interviewee and do not necessarily reflect the views of Future Medicine Ltd (Future Science Ltd).

\section{References}

1 Takashima K, Inoue Y, Tashiro S, Muto K. Lessons for reviewing clinical trials using induced pluripotent stem cells: examining the case of a first-in-human trial for age-related macular degeneration. Regen. Med. 13(2), 123-128 (2018).

2 Nature Editorial. Japan should put the brakes on stem-cell sales. Nature 565(7741), 535-536 (2019).

3 Cyranoski D. Japan's approval of stem-cell treatment for spinal-cord injury concerns scientists. Nature 565(7741), 544-545 (2019). 